\title{
THE SIGNATURE AND G-SIGNATURE OF MANIFOLDS WITH BOUNDARY
}

\author{
CHUAN-CHIH HSIUNG
}

\section{The signature theorem}

Let $M$ be a compact oriented $C^{\infty}$ manifold ${ }^{1}$ of dimension $4 k$ with boundary $B$ of dimension $4 k-1$. The oriented $B$ is called a reflecting boundary of $M$ if it admits an orientation-reversing involution $\pi$. A simple example of the reflecting boundaries of $M$ is a $(4 k-1)$-sphere. For convenience and simplicity, we shall always denote such as $(B, \pi)$ a reflecting boundary with its involution together but with its dimension omitted. The following problem seems to be of interest: Is there any manifold $M$ with a reflecting boundary $(B, \pi)$ on which the involution $\pi$ cannot be extended to the interior of the manifold $M$ ?

Now let $\tilde{M}$ with boundary $\tilde{B}$ be a $C^{\infty}$ homeomorphic copy of $(M, B)$ with the same orientation, and $\mu$ be the homeomorphism so that $\mu(M, B)=(\tilde{M}, \tilde{B})$. Then we can define the double of $M$ with a reflecting boundary $(B, \pi)$ to be a $C^{\infty}$ closed oriented manifold $N$ such that $N=M \cup \tilde{M}$ and that $\tilde{B}=\pi(B)$ by identifying $\mu \pi(x) \in \tilde{B}$ with $x$ for all $x \in B$. Thus on the double $N$ we can define a homeomorphism $\nu: N \rightarrow N$ by:

$$
\nu(x)= \begin{cases}\mu(x), & \text { for } x \in M, \\ \mu^{-1}(x), & \text { for } x \in \tilde{M} .\end{cases}
$$

To see that this is well-defined, at first we notice that $\nu(x)=\mu(x) \in \tilde{B}$ for $x \in \tilde{B}$. Since $x$ is identified with $\mu \pi(x) \in \tilde{B}, \nu(x)=\nu(\mu \pi(x))=\mu^{-1}(\mu \pi(x))=\pi(x) \in B$, and therefore $\mu(x)$ is identified with $\pi(x)$; this is indeed true by the definition of our identification and the assumption $\pi^{2}=1$. Clearly, $\nu$ is an involution. (It should be noted that the definition of doubling a manifold $M$ here is somewhat different from the ordinary one under which $M$ and $\tilde{M}$ are of opposite orientations so that every point of $B$ is a fixed point under the involution $\nu$; for the latter see, for instance, [3].) Alternatively, we may regard doubling the manifold $M$ with a reflecting boundary $(B, \pi)$ as finding a $C^{\infty}$ homeomorphism

$$
h: M \rightarrow N
$$

Communicated February 3, 1972, and, in revised form, June 25, 1972. Research partially supported by the National Science Foundation grant GP-33944.

1 Throughout this paper all manifolds are differentiable. 
where $N$ is a $C^{\infty}$ closed manifold with an involution

$$
\nu: N \rightarrow N
$$

such that $\nu h=h \pi: B \rightarrow N$. Assume $h$ maps $M$ into a fundamental domain of the involution $\nu$ in such a way that $B$ is mapped onto itself. We shall identify $M$ with the fundamental domain henceforth, so that we may regard the double $N$ as composed of two halves $M$ and $\tilde{M}$ with the same orientation such that $M$ is mapped onto $\tilde{M}$ by $\nu, M \cap \tilde{M}=B$, and $\nu \mid B=\pi$.

A Riemannian metric on the double $N$, for which the involution $\nu$ is an isometry, is said to be symmetric, and the restriction of a symmetric metric on $N$ to $M$ is also called a symmetric metric on $M$. Now there arises the problem of deriving a $C^{\infty}$ symmetric Riemannian metric on $N$ from a $C^{\infty}$ Riemannian metric on $M$. For this problem at first we are naturally tempted to prolong to $N$ a differentiable metric $g$ on some manifold containing $M$ by setting

$$
g(x)=g(\nu(x))
$$

for $x \in M$. Although (1.2) is well defined, the difficulty is that the resulting metric will, in general, not be differentiable across $B$. However, on the other hand, for a given $C^{\infty}$ Riemannian metric $g$ everywhere defined on $N$ we may obtain from it a $C^{\infty}$ symmetric metric $g$ by setting

$$
g(x)=\frac{1}{2}[g(x)+g(\nu(x))], \quad \text { for } x \in N .
$$

Now we consider a $C^{\infty}$ symmetric Riemannian metric $g$ on $N$. Then by Hirzebruch signature theorem [5] the signature of $N$ is given by

$$
\operatorname{sign}(N)=\int_{N} L_{k}\left(p_{1}, \cdots, p_{k}\right)\left(\Omega_{g}\right),
$$

where $p_{j}$ is the $j$-th Pontrjagin class of $N$, and $\left\{L_{k}\left(p_{1}, \cdots, p_{k}\right)\left(\Omega_{g}\right)\right\}$ is the Hirzebruch's multiplicative sequence of polynomials with each $p_{i}$ expressed in terms of the curvature 2-forms $\Omega_{j k}$ of the Riemannian metric $g$ by a theorem of Chern [2]. However,

$$
\begin{aligned}
\int_{N} L_{k}\left(p_{1}, \cdots, p_{k}\right)\left(\Omega_{g}\right) & =\left(\int_{M}+\int_{\tilde{M}}\right) L_{k}\left(p_{1}, \cdots, p_{k}\right)\left(\Omega_{g}\right) \\
& =2 \int_{M} L_{k}\left(p_{1}, \cdots, p_{k}\right)\left(\Omega_{g}\right),
\end{aligned}
$$

since $L_{k}\left(p_{1}, \cdots, p_{k}\right)\left(\Omega_{g}\right)$ depends only on the Riemmanian metric $g$, and the metric $g$ is symmetric.

On the other hand, the following theorem was first observed by S. P. Novikov and proved jointly by Atiyah and Singer [1, Prop. (7.1)]: 
Theorem 1.1. Suppose that two compact oriented manifolds $M_{1}$ and $M_{2}$ have a common boundary $B$ with opposite orientations. Then

$$
\operatorname{sign}\left(M_{1} \cup M_{2}\right)=\operatorname{sign}\left(M_{1}, B\right)+\operatorname{sign}\left(M_{2}, B\right),
$$

where sign $\left(M_{i}, B\right)$ denotes the signature of the manifold $M_{i}$ with boundary $B$ for $i=1,2$.

By applying Theorem 1.1 to our case we obtain

$$
\operatorname{sign}(N)=\operatorname{sign}(M, B)+\operatorname{sign}(\tilde{M}, B)=2 \operatorname{sign}(M, B),
$$

since $M$ and $\tilde{M}$ are homeomorphic with the same orientation. Combination of (1.4), (1.5), (1.7) thus gives

$$
\operatorname{sign}(M, B)=\int_{M} L_{k}\left(p_{1}, \cdots, p_{k}\right)\left(\Omega_{g}\right) .
$$

Hence we arrive at

Theorem 1.2. The signature of a compact oriented $C^{\infty}$ manifold $M$ of dimension $4 k$ with a reflecting boundary $B$ of dimension $4 k-1$ is given by (1.8) where $g$ is a symmetric metric on $M$.

When $B$ is empty, Theorem 1.2 reduces to Hirzebruch signature theorem.

\section{The $G$-signature and signature-defect}

We first state the following theorem of Atiyah and Singer [1, p. 588], which is a generalization of Theorem 1.1 on the additivity property of the signature:

Theorem 2.1. Suppose that two compact oriented manifolds $M_{1}$ and $M_{2}$ have a common boundary $B$ with opposite orientations, and that a compact Lie group acts differentiably on $M_{1}$ and $M_{2}$ preserving the orientations. Then

$$
\operatorname{sign}\left(G ; M_{1} \cup M_{2}\right)=\operatorname{sign}\left(G ; M_{1}, B\right)+\operatorname{sign}\left(G ; M_{2}, B\right),
$$

where sign $\left(G ; M_{1} \cup M_{2}\right)$ and $\operatorname{sign}\left(G ; M_{i}, B\right)$ denote, respectively, the $G$ signatures of $M_{1} \cup M_{2}$ and $M_{i}$ with boundary $B$ for $i=1,2$.

As in $\S 1$ let $M$ be a compact oriented manifold of dimension $2 l$ with a reflecting boundary $(B, \pi)$, and $N=M \cup \tilde{M}$ be the double of $M$ with an involution $\nu$ defined by (1.1). Then we naturally intend to extend any automorphism $g$ of $M$ to an automorphism of $N$ by

$$
g(x)= \begin{cases}g(x), & \text { for } x \in M, \\ \nu\left(g \nu^{-1}(x)\right), & \text { for } x \in \tilde{M},\end{cases}
$$

so that

$$
g(\nu \pi(x))=\nu g \nu^{-1}(\nu \pi(x))=\nu g \pi(x) \quad \text { for } x \in B .
$$

On the other hand, for $x \in B$ since $x=\nu \pi(x)$ by our identification for the reflecting boundary $(B, \pi)$, we have 


$$
g(x)=\nu \pi g(x),
$$

which, together with (2.3), implies immediately that to well define an automorphism of $N$ by (2.2) it is necessary that

$$
\pi g=g \pi \quad \text { on } B .
$$

Now let $M$ be a compact oriented manifold of dimension $2 l$ without boundary, and suppose that there is a compact Lie group $G$ acting on $M$ preserving the orientation. For expressing sign $(g ; M)$ for an element $g$ of $G$, in [1] Atiyah and Singer obtained the $G$-signature Theorem (6.12), Corollary (6.13), Proposition (6.15), Corollary (6.16) (which was proved by Conner and Floyd [4, Cor. (27.4)] by a different method), and Proposition (6.18). If $M$ has a reflecting boundary $(B, \pi)$, by using our method of doubling a manifold in $\S 1$ and Theorem 2.1, we can easily show that the above mentioned expressions of Atiyah and Singer also hold for sign $(g ; M, B)$ provided that on the boundary $B, g$ has no fixed point and satisfies the condition (2.5).

Very recently Hirzebruch [6] defined the signature-defect of a finite group acting effectively on a connected compact oriented manifold $M$ without boundary, and obtained some interesting relationships between number theory and the signature-defect at some special points of a four-dimensional $M$.

Now let $M$ be a connected compact oriented manifold of dimension 4 with a reflecting boundary $(B, \pi)$, and suppose that there is a finite group $G$ acting by orientation-preserving diffeomorphisms effectively on $M$ and freely on $B$ such that $\pi G=G \pi$ on $B$. By following Hirzebruch we can easily generalize his definition of the signature-defect of a group action on a manifold without boundary to the $G$-action on $M$, and show that his relationships [6, §5] between number theory and the signature-defect also hold for the signature-defect of the $G$-action on the manifold $M$ with boundary $(B, \pi)$ by using the conditions of $G$ on $(B, \pi)$ and the extention in $\S 1$ of Proposition (6.18) of Atiyah and Singer [1].

\section{References}

[1] M. F. Atiyah \& I. M. Singer, The index of elliptic operators. III, Ann. of Math. 87 (1968) 546-604.

[2] S. S. Chern, On curvature and characteristic classes of a Riemannian manifold, Abh. Math. Sem. Univ. Hamburg 20 (1956) 117-126.

[3] P. E. Conner, The Neumann's problem for differential forms on Riemannian manifolds, Mem. Amer. Math. Soc. No. 20, 1956.

[4] P. E. Conner \& E. E. Floyd, Differentiable periodic maps, Springer, Berlin, 1964.

[5] F. Hirzebruch, Topological methods in algebraic geometry, 3rd ed., Springer, Berlin, 1966.

[6] - The signature theorem: Reminiscences and recreation, Prospects in Math., Annals of Math. Studies, No. 70, Princeton University Press, Princeton, 1971, 3-31.

LEHIGH UNIVERSITY

UNIVERSITY OF WARWICK 